\title{
Human Factor Based Leadership: Critical Leadership Tools to Reduce Burnout and Latent Error in a Time of Accelerating Change
}

\author{
Michael R. Privitera \\ Medical Faculty and Clinician Wellness Program, University of Rochester Medical Center, Rochester, NY, USA \\ Email: Michael_Privitera@urmc.rochester.edu
}

How to cite this paper: Privitera, M.R. (2019) Human Factor Based Leadership: Critical Leadership Tools to Reduce Burnout and Latent Error in a Time of Accelerating Change. Health, 11, 1224-1245. https://doi.org/10.4236/health.2019.119095

Received: August 24, 2019

Accepted: September 27, 2019

Published: September 30, 2019

Copyright $\odot 2019$ by author(s) and Scientific Research Publishing Inc. This work is licensed under the Creative Commons Attribution International License (CC BY 4.0).

http://creativecommons.org/licenses/by/4.0/

\begin{abstract}
The majority of errors in healthcare are from systems factors that create the latent conditions for error to occur. The majority of occupational stressors causing burnout are also the result of systemic factors. Advances in technology create new levels of stress and expectations on healthcare workers (HCW) with an endless infusion of requirements from multiple authoritative sources that are tracked and monitored. The quality of care and safety of patients is affected by the wellbeing of HCWs who now practice in an environment that has become more complex to navigate, often expending limited neural resource (brainpower) on classifying, organizing, constantly making decisions on how and when they can accomplish what is required(extraneous cognitive load) in addition to direct patient care. New information demonstrates profound biological impact on the brains of those who have burnout in areas that affect the quality and safety of the decisions they make-which affects risk to patients in healthcare. Healthcare administration curriculum currently does not include ways to address these stress-induced problems in healthcare delivery. The science of human factors and ergonomics (HFE) promotes system performance and worker wellbeing. Patient safety is one component of system performance. Since many requirements come without resource to accomplish them, it becomes incumbent upon health system leadership to organize the means for completion of these to minimize the needless loss of brain power diverted away from the delivery of patient care. $\mathrm{Hu}$ man Factor-Based Leadership (HFBL) is an interactive, problem solving seminar series designed for healthcare leaders. The purpose is to provide relevant human factor science to integrate into their leadership and management decisions to make HCWs occupational environment more manageable and sustainable-which makes safer conditions for clinician wellbeing and patient care. After learning the content, a cohort of healthcare leaders believed that adequately addressing HFE in healthcare delivery would significantly reduce
\end{abstract}


clinician burnout and risk of latent errors from upstream leadership decisions. An overview of the content of the seminars is described. Leadership feedback on usability of these seminars is reported. Three HFBL seminars described are Human Factor Relevance in Leadership, Biopsychosocial Approach to Wellness and Burnout, Human Factor Based Leadership: Examples and Applications.

\section{Keywords}

Leadership, Burnout, Latent Conditions, Latent Error, Patient Safety, Quality of Care, Human Factor Science, Cognitive Load, Occupational Stress, Work Environment, Healthcare

\section{Introduction}

The Institute of Medicine (IOM) 1999 Report "To Err is Human: Building a Safer Health System" emphasized that the majority of errors in healthcare are the result of systems factors [1]. Similarly, the majority of occupational stressors causing burnout are also the result of systemic factors [2]. Stress at work has been increasing over the last decades as measured by the same instrument over time [3]. The rate of technology advances is accelerating and out of pace with human adaptation [4]. The current healthcare occupational environment continues to create adverse conditions which cause \& perpetuate burnout where clinicians and patients are continually at risk, yet we do not prepare healthcare leaders on how to integrate knowledge of these factors into how they lead.

Many of the well-intended interventions to improve specific elements of quality safety or value, when taken in total, paradoxically are contributing to healthcare system dysfunction, by means of cumulative effect and at times thwarting good care [5]. Clinicians are having to constantly juggle when and how to fit in multiple metrics, regulations, laws, educational mandates, etc. from a multitude of different authorities in healthcare that may not communicate with each other to be aware of the total expectation imparted. Understanding how essential brain power is drained from processing information that is extraneous to the clinical care of the patients helps leaders apply these concepts in different clinical settings. The importance of being able to anticipate how this extraneous cognitive load then affects next clinical decisions made, quality and safety of decisions, personal toll on the clinician and sustainability of practice has been sorely underappreciated and yet is modifiable as a safety risk factor.

Human factor/Ergonomics (HFE) science is the ideal framework to apply in healthcare delivery. The purpose of HFE is to optimize human well-being and overall system performance. Patient safety is one component of system performance [6].

\section{Application of Human Factors/Ergonomics in Healthcare Leadership and Management}

Often HFE is applied to physical work environments work flows, human-tech- 
nology screen interfaces, surgical safety checklists, designing safety into medical devices, etc. In this paper, the intervention method is teaching an application of HFE to healthcare leaders that is pre-clinician, i.e., taught to healthcare leaders, managers and quality and safety professionals to empower them to create better work conditions. Subsequently, this education promotes wellbeing of those they lead while minimizing latent errors and a burnout-inducing environment that could result from their decisions.

The ultimate goal of this Human Factor-Based Leadership (HFBL) curriculum is to improve healthcare system performance, patient safety and staff wellbeing. The content includes HFE principles that can be applied in multiple settings that factor in staff capabilities and limitations more realistically in our current healthcare job descriptions, while applying concepts that create greater efficiency and sustainability.

The initial messaging of this leadership-based intervention is to make clear how their future leadership translates to quality of care, patient safety, patient experience, team functioning as well as recruitment and retention of highly skilled staff which enhances the reputation, net institutional knowledge and performance of the institution.

Figure 1 illustrates how leadership affects key issues in the delivery of highquality care. The figure represents many concepts covered in Human Factor-Based Leadership curriculum. It provides mechanisms on how ultimate patient experience and outcomes get affected by decisions that affect clinicians and their work environment which then affects the healing environment for patients.

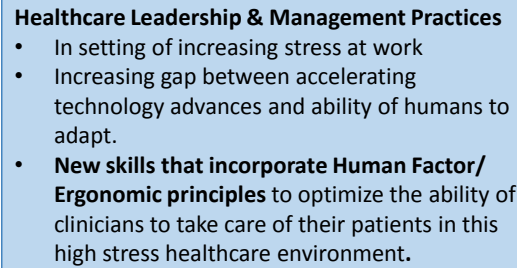

Leadership effect on patient care delivery [Mechanism below:]
Healing Environment Patients Quality of care delivered Patient safety Patient experience Cost containment Retention of skilled staff and institutional knowledge* Better patient access to care Patient loyalty to institution.

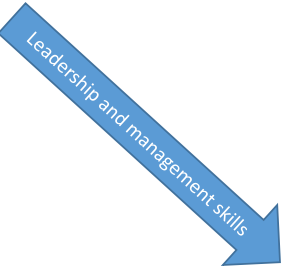

\begin{tabular}{|l|}
\hline \multicolumn{1}{|c|}{ Work Envifonment } \\
\hline Clinicians \\
Wellbeing and safety of staff \\
Clinician engagement and retention \\
Going the extra mile \\
Ability to provide compassionate care \\
Intrinsic joy and meaning in work \\
Loyalty to and trust in institution. \\
\hline
\end{tabular}

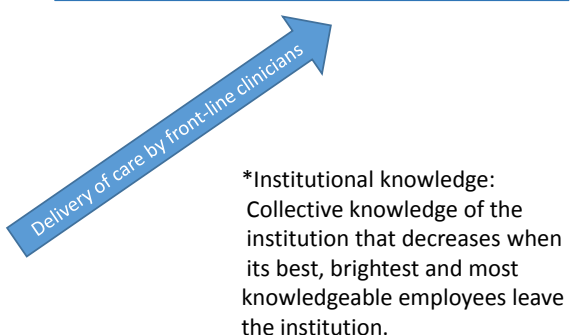

Figure 1. Summary concepts of human factor-based leadership series.

\section{Key Background Facts for Human Factor-Based Leadership Seminar Series}

As external stress and increased job expectations increase, there is essentially no 
discretionary, flexible time left in clinician's work day and often work tasks bleed into what was supposed to be clinician off time. Since these increased demands affect the healthcare worker's ability to stop, rest, recuperate and eat properly, leaders must pay attention that basic human needs in Maslow's hierarchy are being met before higher level brain function on the job can occur [7]). Burnout increases the higher percent of the clinical component of person's healthcare position. However, the most meaningful work for clinicians is patient care [8]. The conclusion from this study was that many factors are getting in the way of clinicians taking care of their patients, wearing them down in a dose-related exposure fashion [8].

Favorable leadership scores are associated with less burnout and increase job satisfaction [9]. Occupational stressors that contribute to burnout per Maslach and Leiter are: Excessive workload physical cognitive and emotional, lack of control, poor balance between effort and reward, lack of community, lack of fairness of resources and justice, value conflict [10]. Excessive workload has often been understood in terms of physical workload, but in today's high expectation healthcare environment, acute and chronic cognitive load becomes relevant in the calculus of human limitations. Examples include: information explosion of medical literature, requirement intrusion into usual clinical workflows, less-than-optimal electronic medical record performance in clinical care, sicker and more medically educated patient population through the internet. Emotion work (e.g., giving bad news to patients and their families, and frustrations involved in getting care for their patients from insurance companies who sometimes use cost control tactics meant to work by wearing down the clinician) becomes highly relevant in the calculus of human limitation as well. A study of New York State physicians showed that of the significant occupational stressors on physicians, $80 \%$ of them are systemic/organizational [2]. As more clinicians are employed instead of private practice, and more decisions in healthcare delivery are made by distant authoritative sources, there is increasing lack of control experienced by clinicians. Poor balance between effort and reward gets worse when more time gets spent upon administrative tasks compared to clinical care of the patient. There is less sense of community of clinicians the more they are isolated off from others by extensive documentation requirements resulting in less interaction with patients and colleagues. Training methods have been increasingly allocated to online learning resources instead of learning in groups with other people. A lack of fairness in allocation of resources and justice is felt by clinicians when hospital resources are being used on satisfying and maximizing externally imposed metrics of quality (even when not evidenced-based), paying for consulting companies that optimize metrics for patient satisfaction, and other resource allocation that gets shifted away from the direct provision of patient care. Value conflicts constantly occur in clinicians when having to spend more time on administrative tasks instead of spending time with their patients, or having to see patients in high-pressure high-volume clinics that process patients through rather than getting to know them and making the best decisions 
on their behalf.

Patient satisfaction is negatively correlated with staff intention to leave the institution [11] and is positively correlated with clinician career satisfaction [12]. Psychosocial safety climate (PSC) is an upstream determinant of multiple work-related conditions, efficiencies and outcomes [13] [14], hence needs to be understood and addressed by the healthcare leader.

\subsection{Implementation of Leadership and Management Series}

Human Factor-Based Leadership Seminar Series included these three seminars:

1) Human Factor Relevance in Leadership;

2) Biopsychosocial Approach to Wellness and Burnout;

3) Human Factor Based Leadership: Examples and Applications.

Seminar attendees were hospital leaders from different disciplines (Table 1).

Table 1. Human factor-based leadership (HFBL) attendee \% composition.

\begin{tabular}{cc}
\hline Physician $58 \%$ & Nurse $19 \%$ \\
Psychologist \&/EdD 10\% & Social Worker 3\% \\
Advanced Practice Provider 3\% & Other 6\% \\
\hline
\end{tabular}

Since leaders are in positions of being problem solvers, it was important to keep seminars practical and applicable. The recommended style of implementation of each seminar was to create two or three stopping points in each module to give the opportunity for leaders to discuss and apply the material to pre-planned questions or scenarios that are posed to them. Attendees worked in small groups and reported out their findings for each group to share learning. Each module was co-taught with another faculty member. Usually one faculty would focus on presentation content, the other would activate and guide the attendees and help lead discussion. 90 minutes was allowed for each module. Post-seminar evaluations were completed by leader attendees. Data from these evaluations was analyzed to determine how seminar content was achieving the aims intended, and assessment as to whether information taught could significantly reduce risk of latent error and reduce clinician burnout.

At the end of each module content description, the result of evaluations of each seminar by hospital leaders will be reported on these three post seminar evaluation questions:

- Today's session on average improved my knowledge of (seminar title: Human Factor Relevance in Leadership or Biopsychosocial Approach to Wellness and Burnout or. Human Factor Based Leadership: Examples and Applications) by $\%$.

○ $\quad 0 \%=$ I already knew all this material.

- $100 \%=$ All this material was new to me.

- What average $\%$ of what was covered today do you expect to be useful to you in your leadership career?

○ $0 \%=$ None of it. 
○ $100 \%=$ All of it.

- On average how much of the information covered today do you think would be useful to healthcare leaders in general?

○ $0 \%=$ None of it.

○ $100 \%=$ All of it.

The study analysis of this data was submitted for review to Institutional Review Board, University of Rochester Medical Center. This study was given not human research determination and there-for exempt status. The basic content of the modules will be presented below in outline form and convey the key facts of the slide presentations.

\subsection{Human Factors/Ergonomics (HFE) Definition}

Ergonomics: An applied science concerned with designing and arranging things that people use so that the people, things and other elements of a system interact most efficiently and safely. The scientific discipline concerned with understanding interactions among humans and other elements of a system-also called human factors, biotechnology, and human engineering [6].

\section{Content Outlines of Three Human Factor-Based Leadership Seminar Series and Evaluations}

\subsection{Module 1: Human Factor Relevance in Leadership}

- How to understand the relationship between leadership, people management practices and the impact on patient care (see Figure 1).

- Definition, purpose, range and goal of Human Factors and Ergonomics in healthcare.

- Three types of ergonomics: physical, neurocognitive, and organizational.

- Three phases of awareness of human factors influence on healthcare workers and therefore their patients: 1) an invisible problem, not see HFE influence; 2) see HFE influence but not acknowledge it; 3) awareness of HFE influence and addressing it as a systemic/organizational issue.

- There is a perfect storm of health care reform history in the setting of Medicine's culture of endurance and silence. The internal psychological environment of the clinician and the immediate external clinical environment are both invalidating to human limitations and human needs of healthcare workers.

- Hospital systems are surrounded by a stormy and tempestuous external environment created by multiple factors that affect internal hospital/healthcare institution culture and operations.

- In the US, an increasing percent of business revenue is going to corporate leadership salaries compared to rank and file workers [15]. Healthcare is profitable and sometimes shareholders are main concern in the business of medicine, yet little awareness of these factors is known to those doing the work of patient care-constantly having to do more with less resource where it is needed [16] [17]. 
- Strategies for cutting costs include downsizing, automation, and outsourcing of jobs to the "customer", who sometimes is the patient and sometimes is the healthcare worker [18].

- Healthcare Failure Mode and Effect Analysis (HFMEA) is a method that can evaluate "shadow work": The unseen, unpaid jobs that fill your day [19]. HFMEA breaks down the clinical workflow into steps that demonstrate what is involved and factors that may have gone wrong or require shadow work at each step. It visually illustrates the difference between prescribed work (what the administrator sees) and real work done (prescribed work plus the shadow work to accomplish the prescribed work).

- While stress at work is increasing nationally and burnout is increasing, over this same time period personnel costs have increased, and technology costs have decreased. This situation leads to the process of disintermediationmoving supportive intermediary staff who used to help with process completion [18]. Online applications for forms, computer-based training for mandatory trainings, etc., have substituted for the previously supportive intermediary staff. Subsequently, more workload is off-loaded to clinician without time or resources to do so, competes with time to see patients, and often bleeds into their off time which should be for their recuperation and family time. Increased frustration occurs from poor electronic medical record operability that contributes to more work outside of work (WOW).

- The impact of clinician burnout has multiple dose-related relationships of impact on institution and the patient: Medical errors, malpractice, disruptive behavior, reduced empathy for patients, decreased patient and career satisfaction, reduced adherence to treatment regimens [20] [21] [22].

- Impact of clinician burnout financially: Drop in patient satisfaction scores, increased turnover, medical claims by employees, short term and long term disability costs [19] [20] [21].

- Impact of clinician burnout personally: Higher suicide rate, substance abuse, divorce, coronary heart disease and depression [22] [23].

- Technology is outpacing human adaptability requiring smarter ways of addressing leadership training, education of clinicians and use of resources [4] [24].

- Human impact of electronic medical record (EMR) and other health technology affordances intruding into home life, family life and ability to recuperate for the next work day has not been factored in with current leadership decisions. The times of day used to do work outside of work (EMR at home) is when fatigue impairments in thinking occur-yet clinicians are clinically and legally responsible for what they order for patients and write in the chart [25] [26] [27] [28].

- Excessive EMR use bleeding into home life on weekends and evenings, increases odds of burnout by $46 \%$, increasing job stress and decreasing job satisfaction [2]. A related study describes the impact of work-home conflict on risk of burnout, depression and seriously contemplating separation or di- 
vorce [29].

- Upstream factors (blunt end of patient care) can create latent conditions for error and burnout at the sharp end of patient care (clinician/patient interface) [30].

- Academic Medical Center operational disadvantage: Hierarchy, managerial filtering of information, gate keeping slows or impairs needed information to get to senior leaders to make optimal decisions. Boots on ground have a fear of feedback sanctions on their career if they speak up.

- Parallel stories of insufficiently addressing systemic factors in production of error and its role in producing burnout are described. The tsunami of well-intended interventions to improve quality, safety or value at the level of the individual clinician is producing health system dysfunction backfiring in intent, and paradoxically creating less safety and quality. Not enough attention has been paid to organizational/systemic solutions for safety and unfortunately too much attention has been paid on what the individual clinician must do to improve patient safety [5].

- Framework for public health action. Health impact pyramid. Increasing population impact the more organizational/systemic the intervention, with increasing individual effort needed and least population impact when emphasis at the individual clinician level [31].

- Listing in one place the total number of mandatory requirements asked of clinicians to do, shows the evidence of how overwhelming the expectations would be to anyone. It is incumbent upon the organizational leadership to take on ways to lessen these compliance pain points, support completion in more creative ways that align with healthcare worker's intrinsic desire to be better at what they do. Decrease chaos effect on patient care and individual wellbeing.

- Creating a mandatory management proposal review and its key components is described. A hospital solution to management and compliance with multiple externally imposed requirements, laws and other mandates becomes a necessity to organize the logistics of completion.

- The usual way human factor application science has been applied to reduce the risk of confusion between choices a clinician has to make in patient care has been by making it easier to make the right choice and harder to make the wrong choice [32].

- New application of human factors science in leadership and management is to reduce the systemic influences on clinical performance, helping leaders be aware of systemic factors at play, with models of leadership approach that mitigates risk of latent error and clinician burnout [30] [33].

- One example of human factor based leadership application: Organizing and supporting the completion of externally imposed mandates, laws regulations and policies.

- Concept of neural resource (brain power, synaptical currency, brain capital) and how important to preserve this resource for the high-level clinical deci- 
sions made in patient care. The goal is to provide enough understanding the cognitive neuroscience to help leaders set up working conditions that optimize the use of brain power needed for clinical decision-making and understanding the risks of not doing so.

- Executive function neural resource is used up in these processes: Focus, decision making sorting classifying, getting back on track after interruptions, maintaining goals, working memory, self-regulation, professionalism, despite how treated by a patient, family member or other staff. Maintaining "aequinimitas" in setting of bleeding, injury pain, etc., and emotion work in dealing with bad outcomes [34] [35].

- New thoughts for leaders to include budgeting of neural resources when also budgeting financial resource, space, staffing, etc. for optimal use of highly trained clinicians brain power. The application of this concept would be to minimize extraneous cognitive load on clinicians.

- Summary of key leadership new material: Optimal workloads (cognitive, emotional, physical), optimal use of brain power. Look beyond time on (salaried at work), and firmly preserve employee time off. Address human needs, restoration, boundary between work and home despite technology affordances that constantly create challenge this boundary. Potential availability to work 24/7, promotes higher sustained tension and need for vigilance even at home. Be aware of shadow work, pain points for employees, and how this affects quality of care they provide, ergonomic application, top of the license work.

\section{Module 1 Evaluations}

- Today's session on average improved my knowledge of Human Factor Relevance in Leadership by: Mean $=47 \%{ }^{\star} \mathrm{N}=26$.

- What average $\%$ of what was covered today do you expect to be useful to you in your leadership career? $80 \% \mathrm{~N}=30$.

- On average how much of the information covered today do you think would be useful to healthcare leaders in general? $92 \%$.

${ }^{*}$ May be affected by attendees having previously been exposed to this material as applied in many wellness initiatives at URMC.

\subsection{Module 2: Biopsychosocial Approach to Wellness and Burnout}

- Biopsychosocial Model of George Engel, hierarchy of natural systems and continuum [36].

- Six categories of work stress that contribute to burnout: Excessive workload of physical, cognitive and emotion work; Lack of control, poor balance between effort and reward; Lack of community; Lack of fairness, value conflict [10].

- Struggle between the emotional brain and the wise brain: Increased amygdala activity and decreased prefrontal cortex activity that can occur; Multiple healthcare examples: Effect of difficult patient, violence, bullying, frustrations, lapses in self-regulation and over-correction, coping with multiple fac- 
tors that thwart good care, emotion work, chronic occupational stress, burnout and depression [37] [38] [39].

- NASA TLX as a metric of workload [38].

- Increased cognitive workload of NASA TLX score correlates with increased risk of medical error.

- Biological changes in burned out individual: Cortisol changes, hyper then hypo secretion, prefrontal cortex thinning. Amygdala enlarges [37] [40] [41] [42]. Hippocampus shrinks [43]. In one year of physician internship DNA's telomeres shorten six times greater than age-matched controls (aging phenomenon) (see Figure 2) [44].

- Depressive and aggressive reactions to stress in burnout are dose-related. The higher the burnout level the higher the depression and aggression [45].

- Rudeness and disruptive behavior in healthcare delivery affect errors, lower quality of care and higher patient mortality [46]. Emphasis should be on improving teamwork culture, safety climate and work-life balance [47].

- Motivation as relevant in healthcare. Motivators in work that has cognitive component to the work are purpose, mastery and autonomy. Money incentive in this setting may backfire on intent [48] [49].

- The higher the burnout severity the higher the odds ratio for having major depression [45] [50].

- Several neurotransmitters are involved in coping with stress. Deficiencies are associated with specific behaviors that get manifested by the clinician especially if becomes clinically depressed [51] [52] [53] [54].

- Recognizing when a colleague or person you supervise is emotionally not doing well was discussed as well as encouraging them to get help.

- How successful leaders think. Integrative thinkers creatively resolve tensions among opposing ideas, creating superior outcomes compared to conventional thinkers who tend to think in linear ways (more A causes more B) but miss unintended consequences that occur by affecting other parts of the ecosystem of healthcare [55].

1. Hormonal: Chronic cortisol changes (hyper then hypo), leads to plaques on coronary arteries.

2. DNA changes: Telomere shortening (cellular aging) in medical interns, 6 times faster than controls-increased risk of numerous medical conditions.

3. Neurotransmitter: excess glutamate decreases grey matter of Basal Ganglia- which decreases fine motor control.

4. Anatomical changes:

a. Thinning of Pre-frontal cortex-affects ability to focus, attention, quality of medical decision-making.

b. Enlargement of Amygdala- creates increased reactivity to stress

c. Hippocampus shrinking- reduces short term memory at 4-6 weeks of high stress, then reduces long term memory if more than 12 weeks high stress.

d. Creates head MRI findings that are similar to early life trauma patients.

Figure 2. Biologic changes in high chronic occupational stress and burned out individual. 
- Finding the therapeutic window for optimal quality and safety when creating and instituting mandates, regulations and laws in the name of patient safety. Past certain amount of information processing, prefrontal cortex shuts down and poor decisions occur [56].

- Cognitive flexible memory accesses what is learned in medical training for applying a differential diagnosis to the patient symptom presentation. Under high stress neural resource used up and thinking converts to habit memory, sparing cognitive resources, automates response to stimulus, leading to a less satisfactory differential diagnosis and care plan [57] [58].

- Cognitive Load Theory. Intrinsic load: Inherent level of load necessary for diagnosis and treatment. Germane load: related to the care of patient like anticipating need for support to patient or family. Extraneous cognitive load: Burden of cognitive processing that can be improved by better design. We need to reduce extraneous cognitive load [59] on clinicians to free their brain power to be able to operate on the thinking level they need to when doing clinical work.

- Healthcare ecosystem-multiple national, state, industry regulatory authorities in healthcare. Many have patient as primary concern. Business of healthcare may have shareholders as primary concern. The combination results in numerous administrative task demands of mandatories, policies, and laws, regulations, quality metrics and billing requirements all affecting workflow. The only control point in this ecosystem is at level of hospital leadership. Ways they can improve the experience of completing all the needed requirements was discussed. Importance of Human Factor based leadership: To apply methods of reduction of extraneous cognitive load to organize the roll out of these expectations on healthcare workers in workable and sustainable way.

- Integrative Model: Patient Safety and Staff Wellbeing. Visual translational model that represents nearly fifty published studies that describe the effect of high acute occupational stress on risk for error and high chronic occupational stress on the brain of the clinician, devolving the quality of their work by means of biological changes of their brain affecting key areas of decision-making and memory. The model illustrates the need to discern between those safety-related barriers that are well designed and reduce the risk of error and those that have appearances of enhancing safety, but may be without evidence, or too many in number that acutely or chronically can thwart good care and subsequently increase risk of error [57].

- Burnout organizational interventions. The most important organizational intervention: Go beyond "The Triple Aim" healthcare delivery framework that focused upon costs, quality and patient experience [60] [61] and expand to a "Quadruple Aim" healthcare delivery framework. This includes the experience of providing care when also making decisions about costs, quality and patient experience [62] [63] [64]. Paying more attention to human limitations in delivery of care and overcoming medical culture of endurance and silence. Leadership style and concern is key. Leadership message of caring 
about wellbeing of employees, commitment to action. If leadership overly delegates to others, the messaging of the importance of the wellness initiative will not be seen by employees as sufficient. Senior leaders should need to give the initial message, align forces in the same direction, monitor regularly and show involvement. Create culture that encourages the boundary between work and home, such that home time is off time without regular work expected to be done [11]. Measure burnout/wellbeing. Establish Wellness initiative strategic planning work group. Understand front line problems. An anonymous survey to learn pain points. Round table discussions to discuss survey findings openly and gather more input for solutions from front-line clinicians. Simplify access points and processes for clinicians to contribute to organizational improvements. Encourage stronger administrator/clinician partnerships. Organize and assist completion of all mandatories, regulations. Encourage clinicians to seek help for stress, burnout or clinical depression as these are treatable. There should be no reporting of seeking mental health care on licensure, malpractice carrier, credentialing applications or renewals. Protection of the public is best served and relevant only if the clinician is currently impaired to practice medicine. Confidentiality in seeking help is critical to the wellbeing of the clinician and the public.

\section{Module 2 Evaluations}

- Today's session on average improved my knowledge of Biopsychosocial Approach to Wellness by: Mean $=50.4 \%^{*} \mathrm{~N}=22$.

- What average $\%$ of what was covered today do you expect to be useful to you in your leadership career? Mean $85.2 \% \mathrm{~N}=22$.

- On average how much of the information covered today do you think would be useful to healthcare leaders in general? Mean $=90 \% \mathrm{~N}=23$.

* May be affected by attendees having previously been exposed to some of the material through application in certain hospital operations.

\subsection{Module 3: Human Factor Based Leadership: Examples and Applications}

- Multiple theories/principles applied to building a wellness program. Business case, conservation of energy, economy of scale, psychosocial safety climate, organizational health, emotional intelligence, cognitive load (decrease extraneous cognitive load), preservation of clinician executive function for clinical purposes, positive behavioral support-(supporting intrinsic compassion, drive for quality of care, altruism, and avoid coercion). Maintain cognitive schema familiarity and operational similarity across an enterprise in IT interfaces-(reduces extraneous cognitive load). Yerkes-Dodson Law (reduce multitasking and split-attention effect). Bottom up impact on work environment, clinician and staff engagement. Esprit de corps. Support Primary Control Coping (fix the problem causing the stress) and Secondary Control Coping (find ways to adjust to residual occupational stressors) [65].

- Ergonomics: Physical, (neuro)cognitive and organizational. 
- Organization-wide stress management program had a $71 \%$ reduction of malpractice claims outcome in one year [66].

- Emotional influences in patient safety are significant and are another reason to reduce the severity of occupational stress [34].

- Error sensitivity to sleep deprivation in burnout and depression [67]. Burned out residents more sensitive to error with sleep deprivation.

- Medical errors cause personal distress, reduced empathy and increased risk of future errors [68].

- There is a cyclic relationship between burnout and errors, perpetuated by organizational contributions to burnout/depression and organizational contributions to medical errors.

- American Medical Association (AMA) Healthy Workplace Study. Improving workflows reduce burnout 6 fold. Targeted quality improvement projects reduce burnout 5 fold. Improving communication between team members improves professional satisfaction 3 fold [69].

- The most conservatrive interpretation of a law, regulation or mandate is not necessarily the safest. Unintended consequences must be considered as the proposed interpretation may affect the safety of another clinical workflow. Clinicians who are closest to the care delivery need to be part of the decisions of interpretation as may help in articulating the potential negative consequence and help in the design of a safe and feasible solution. Due consideration must be made for human limitations and whether the total expectations on the clinician are feasible or counterproductive.

- Strategies designed to prevent individual error, grounded in system-based cognitive psychological research. Reducing workplace fatigue and stress, reliance on memory, reliance on vigilance, manual calculations. Design and redesign useful polices, standardized unit practices based on evidence [70] [71].

- Strong forces discourage clinician self-care and speaking up. A collage was presented that described frequent clinician internal scripts and doubts, with external scripts from immediate clinical environment invalidating the notion of clinician human limitation.

- Genesis of a Mandate. From bad behaviors or mistakes with bad outcomes, attempts at remedy in the form of laws and policies. "Training" is often asolution somewhere in the law or policy. Example of a New York State law “... requires that all employers operating in New York have a sexual harassment prevention policy and train all their employees" [72]. It then becomes a person's responsibility at institution level to make sure this mandate is satisfactorily implemented and must account to their leadership and the regulators. Mandate education companies have now thrived in this environment as they offer ready-made training solutions and advertise about "surpassing" requirements, which sounds attractive to an administrator in a silo. However there usually is no centralized institutional accounting for all the other staff mandates that are required, the time they take nor the logistics on how these are accomplished in the setting of their clinical duties. Length, content of 
training and options for method of training are all in the organization's control. Administrative decisions on these factors have more impact on clinicians' capability to do their entire job than has often been recognized.

- One institution attempt at creating a composite list of mandates by researching relevant institutional authorities came up with 38 mandates. Using other sources involved in institution education their total of institutional mandates was 84 though have some overlap.

- There needs to be integrated mechanisms in the institution that protect clinicians from unnecessary high level occupational stress by institutionally taking on the responsibility to inventory all mandates and create a centralized process to manage their content creation and roll out logistics, sparing clinicians from extraneous cognitive load, wasted time or effort and preserving their brain power for patient care (Figure 3).

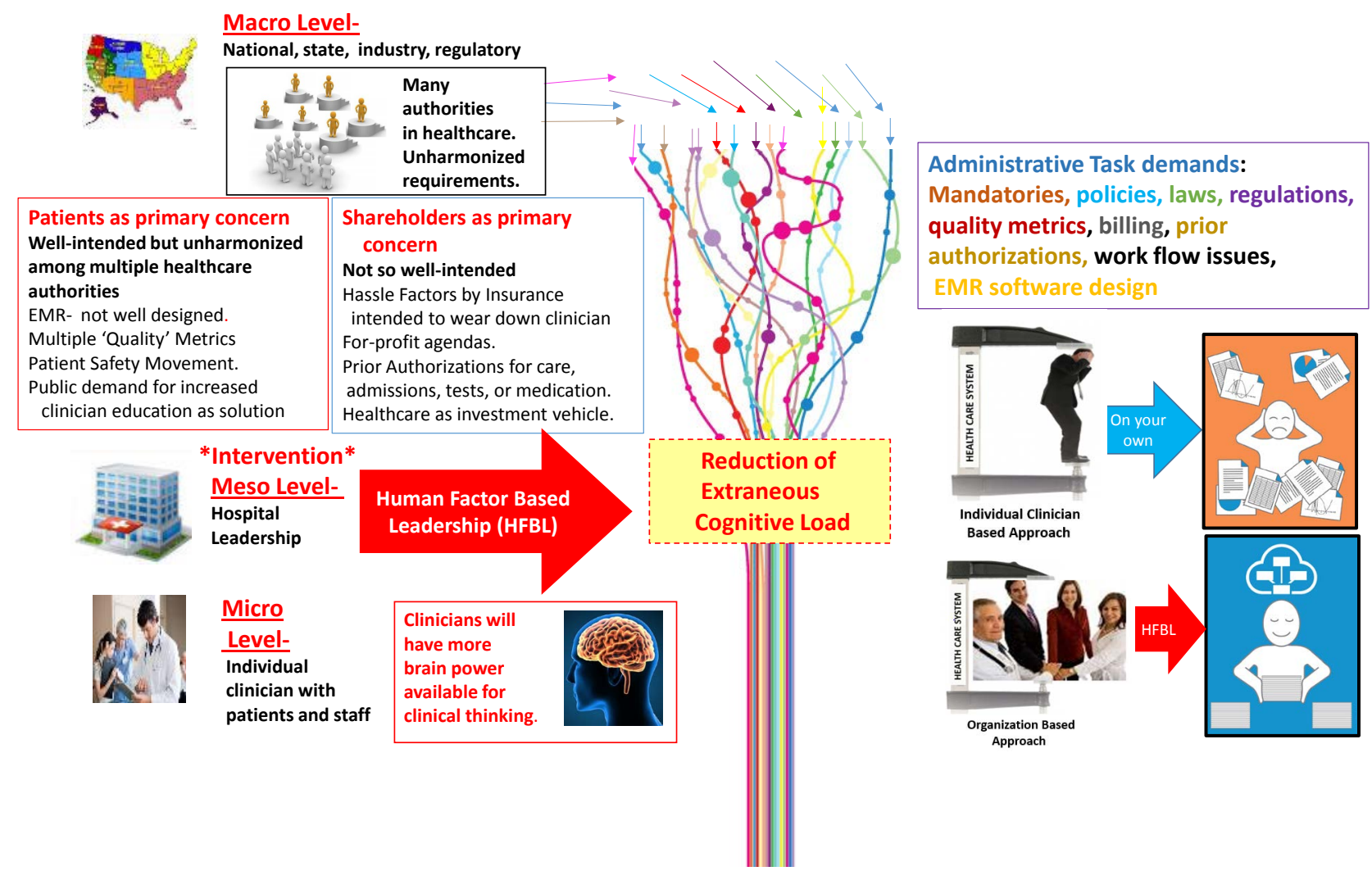

Figure 3. Healthcare ecosystem. Macro Level: Large scale driving forces in healthcare that occur at national, state or local authoritative levels; some with patients as primary concern and well-intended and some from the business of medicine with healthcare company shareholders as primary concern; Meso Level: Forces of control are hospital and other healthcare organization leadership that administer healthcare through their processes, structures and healthcare workers; Micro Level: Forces are at the front line of healthcare delivery including the interaction between clinicians, patients, families and other healthcare staff. The proposed level of intervention of this Human Factor-Based Leadership seminar series is at the Meso level, where hospital leaders have a level of control on how various mandatories, administrative tasks that clinicians need to do to get achieved. Leaders control distribution of resources to help Micro level activity of healthcare delivery to successfully achieve their occupational goals. This seminar series is meant to help prepare hospital leaders to better understand, anticipate, prevent and reduce the factors that interfere with achieving efficient and compassionate healthcare delivery and that create conditions for distress, error and burnout at the point of care. 
- A form of organizational ergonomics application is centralized mandate management.

- Proposed mandate solutions: Single institutional committee to track and advise on content, possible methods of completion, and manage the tendency for "compliance creep" (locally increasing the compliance requirement to more than was intended by the authority source).

- Satisfice mandatory portion ("satisfice" = satisfactory and sufficient to meet requirement but no extras) [73], giving additional learning opportunity for those interested. Improving the experience of completing mandatory requirements by engaging intrinsic desire to learn and give good care. More effective learning methods are needed. Avoid inflammatory, controlling language. Allow mass completion of mandates (as a group-meeting, grand rounds, etc.). Need options beyond individual computer-based online training on their own. Consider employee input into methods to accomplish mandates where possible.

- Avoid short-term lower-cost solutions to mandated education such as prepackaged training company products which may not have the intention to keep content concise and efficiently learned. Be aware that a universal fallacy of increasing expectations on clinicians is underestimation of negative impact as each is silo-ed and separately thought to be minimal inconvenience. Hence, we must keep track of total expectations. Keep mandate lists in one place to see total expectations. Keep in mind of unintended consequences when classifying something as mandatory. Since object of mandate is to demonstrate knowledge of the subject, allow "testing out" so staff can go directly to questions. Keep in mind resource of employee time, and brain power, and should be reframed as a cost of doing business expense. Time to accomplish mandates during the work day must be offered, which creates an economic force to encourage mandates to be made shorter and to the point, as well as software used to be more efficient to complete. Create enduring educational material of content such that clinicians can call up the information in the future as clinically needed.

- Institute for Healthcare Improvement's: "Breaking the Rules for Better Care". Multiple IHI Alliance hospitals asked patients families, clinical and non-clinical staff: If you could break or change any rule in service of a better care experience for patients or staff, what would it be?" [74].

- From twenty-two IHI Alliance participating organizations, they identified 342 rules perceived to provide little or no value to patients and staff. They were classified as habits, organization-specific and actual statutory and regulatory requirements. $78 \%$ were fully within administrative control to change.

- Four types of action on these rules: Debunk myths about nonexistent or misinterpreted rules through staff education; seek clarity from appropriate regulatory agency on true scope and intent of rules; change local administrative polices for which no sufficient rationale was found; speak with collective voice to policy makers about regulations that are harmful to the care or 
wasteful of limited resources.

- For better clinician/Administrator relationships, responsibilities for clinicians and for administrators need to be understood. Merlino's Responsibility Matrix outlines the responsibilities of both clinicians and administrators, which help promote improved relationships between both. Clinicians must acknowledge change, own safety and quality, promote accountability and peer mentoring, stop bad behavior, practice humility and lead by example. Administrators must validate suffering, communicate, and help clinicians understand the business, be inclusive, recognize the need for symbiosis and beware of trigger issues [75].

\section{Module 3 Evaluations}

- Today's session on average improved my knowledge of Human Factors Based Leadership Examples and Applications by: Mean $=62 \% \mathrm{~N}=20$.

- What average \% of what was covered today do you expect to be useful to you in your leadership career? Mean $87.2 \% \mathrm{~N}=18$.

- On average how much of the information covered today do you think would be useful to healthcare leaders in general? Mean $=91.1 \% \mathrm{~N}=18$.

- What type of healthcare leaders would this be most useful to? Write in responses, some more than once: Nursing leaders, administrators, physicians, nurse managers, those in leadership, C-suite, any and all, human resources, quality and safety staff and leaders, leadership at the top of the organization, the dean, chiefs of service, CEO, CFO, leadership who set the culture.

\subsection{Overall HFBL Seminar Series Key Findings on Anticipated Patient Outcomes, Burnout and Latent Error}

1) Could inadequately addressing HFE in healthcare delivery potentially have a negative impact on patient outcomes? 95\% (20) Yes; 5\% (1) No.

2) Would adequately addressing HFE in healthcare delivery significantly reduce burnout in clinicians? $100 \%$ (21) Yes; $0 \%(0)$ No.

3) Would adequately addressing HFE in healthcare delivery significantly reduce risk of Latent Error (Latent Conditions) in healthcare systems?

$100 \%$ (21) Yes; 0\% (0) No.

\section{Conclusion}

Healthcare decision-makers need new tools to manage, educate, lead and deploy limited resources in a rapidly changing healthcare environment. Human Factor Based leadership curriculum is proposed to help fill this current gap in leadership fund of knowledge. There is a negative impact on clinicians and their patients by not addressing these issues. Technology with consequent expectations is changing more rapidly than is human adaptation. Clinician neural resource (brain power) needs budgeting in its use and the ability to recharge by down time. Clinician's off duty time must be respected and actively protected as technology affordances have increasing blurred boundaries between work and home 
life. The technological ease of being able to work at home during off time is mentally tethering the clinician to work issues for unnaturally prolonged times of vigilance, which does not factor in human needs, human limitations nor the unintended negative consequences that follow to patients and clinicians.

\section{Acknowledgements}

The author would like to express his gratitude to many people who helped make this project possible. Educational efforts at the University of Rochester Medical Center were in collaboration with Janine Shapiro MD, Associate Dean of Faculty Development Office for Human Factor-Based Leadership (HFBL) series which had a total of 6 modules for hospital leaders. The three HFE modules described in this paper were part of this series plus:

1) Institute for Healthcare Improvement (IHI) Framework of Improving Joy in Work. Our emphasis was on removing occupational stressors to allow intrinsic joy of taking care of patients to come through.

2) Self-Determination Theory and Work Related Outcomes and

3) Participatory Management Models-Application in your department or division.

Sarah Peyre EdD, Director of the Institute for Innovative Education, collaborated on sponsoring the three HFE modules for presentation to quality and safety professionals whose series was entitled "Integrated Model" Patient Safety and Clinician Wellbeing" as well as video recording the three modules. If want more information on these videos, contact URMC Institute for Innovative Education through website:

https://www.urmc.rochester.edu/institute-innovative-education/history.aspx.

The author received a Patient Safety Award grant for one year from our malpractice carrier MCIC of Vermont to help support this project of preparing and teaching these seminars. The author would also like to thank Michael Apostolakos MD, Chief Medical Officer, Vice President of University of Rochester Medical Center and Robert Panzer MD, Chief Quality Officer and Associate Vice President for, Patient Care Quality \& Safety at University of Rochester Medical Center who helped champion this project for receiving the Patient Safety Award from MCIC.

\section{Conflicts of Interest}

The author declares no conflicts of interest regarding the publication of this paper.

\section{References}

[1] Kohn, L.T., Corrigan, J. and Donaldson, M.S. (2000) To Err Is Human: Building a Safer Health System. Institute of Medicine, National Academy of Sciences, Washington DC.

[2] Privitera, M.R., Attalah, F., et al. (2018) Physicians' Electronic Health Record Use at 
Home, Job Satisfaction, Job Stress and Burnout. Journal of Hospital Administration, 7, 52-58. https://doi.org/10.5430/jha.v7n4p52

[3] Cohen, S. and Janicki-Deverts, D. (2012) Who's Stressed? Distributions of Psychological Stress in the United States in Probability Samples from 1983, 2006, and 2009. Journal of Applied Social Psychology, 42, 1320-1334. https://doi.org/10.1111/j.1559-1816.2012.00900.x

[4] Friedman, T. (2016) Thank You for Being Late: An Optimist's Guide to Thriving in the Age of Accelerations. Farrar, Straus \& Giroux, New York.

[5] Sinsky, C.A. and Privitera, M.R. (2018) Creating a "Manageable Cockpit" for Clinicians: A Shared Responsibility. JAMA Internal Medicine, 178, 741-742. https://doi.org/10.1001/jamainternmed.2018.0575

[6] International Ergonomics Association. http://www.iea.cc/

[7] Maslow, A. and Lowery, R., Eds. (1998) Toward a Psychology of Being. 3rd Edition, John Wiley \& Sons, New York.

[8] Anandarajah, A., Quill, T. and Privitera, M.R. (2018) Adopting the Quadruple Aim: The University of Rochester Medical Center Experience. Moving from Physician Burnout to Physician Resilience. American Journal of Medicine, 131, 979-986. https://doi.org/10.1016/j.amjmed.2018.04.034

[9] Shanafelt, T.D., Gorringe, G., et al. (2015) Impact of Organizational Leadership on Physician Burnout and Satisfaction. Mayo Clinic Proceedings, 90, 432-440. https://doi.org/10.1016/j.mayocp.2015.01.012

[10] Maslach, C. and Leiter, M.P. (1997) The Truth about Burnout: How Organizations Cause Personal Stress and What to Do about It. Jossey-Bass, San Francisco, CA.

[11] West, M., Dawson, J., et al. (2011) Employee Engagement and NHS Performance. NHS Staff Management and Health Service Quality. Department of Health, UK Government, London.

[12] DeVoe, J., Fryer, G.E., Straub, A., McCann, J. and Fairbrother, G. (2007) Congruent Satisfaction. Is There Geographic Correlation between Patient and Physician Satisfaction? Medical Care, 45, 88-94. https://doi.org/10.1097/01.mlr.0000241048.85215.8b

[13] McLinton, S.S., Dollard, M.F. and Tuckey, M.R. (2018) New Perspectives on Psychosocial Safety Climate in Healthcare: A Mixed Methods Approach. Safety Science, 109, 236-245. https://doi.org/10.1016/j.ssci.2018.06.005

[14] Law, R., Dollard, M.F., Tuckey, M.R. and Dormann, C. (2011) Psychosocial Safety Climate as a Lead Indicator of Workplace Bullying and Harassment, Job Resources, Psychological Health and Employee Engagement. Accident Analysis \& Prevention, 43, 1782-1793. https://doi.org/10.1016/j.aap.2011.04.010

[15] Institute for Policy Studies (2018). https://ips-dc.org/

[16] Haefner, M. (2017) Aetna's Profits Rise 39\% Despite Revenue Shortfall. https://www.beckershospitalreview.com/payer-issues/aetna-s-profits-rise-39-despite -revenue-shortfall.html

[17] Gooch, K. (2019) Positive Outlook Seen for US Healthcare Stocks This Year. Becker's Hospital Review.

https://www.beckershospitalreview.com/finance/positive-outlook-seen-for-us-healt hcare-stocks-this-year.html

[18] Lambert, C. (2015) Shadow Work: The Unseen, Unpaid Jobs That Fill Your Day. Counterpoint Press. 
[19] HFMA.

https://www.cms.gov/Medicare/Provider-Enrollment-and-Certification/QAPI/dow nloads/GuidanceForFMEA.pdf

[20] Panagioti, M., Geraghty, K., et al. (2018) Association between Physician Burnout and Patient Safety, Professionalism, and Patient Satisfaction. JAMA Internal Medicine, 178, 1317-1331. https://doi.org/10.1001/jamainternmed.2018.3713

[21] Anagnostopoulos, F., Liolios, E., et al. (2012) Physician Burnout and Patient Satisfaction with Consultation in Primary Health Care Settings: Evidence of Relationships from a One-with-Many Design. Journal of Clinical Psychology in Medical Settings, 19, 401-410. https://doi.org/10.1007/s10880-011-9278-8

[22] Toker, S., Melamed, S., Berliner, S., Zeltser, D. and Shapira, I. (2012) Burnout and Risk of Coronary Heart Disease: A Prospective Study of 8838 Employees. Psychosomatic Medicine, 74, 840-847. https://doi.org/10.1097/PSY.0b013e31826c3174

[23] Ahola, K., Hakanen, J., Perhoniemi, R. and Mutanen, P. (2014) Relationship between Burnout and Depressive Symptoms: A Study Using the Person-Centered Approach. Burnout Research, 1, 29-37. https://doi.org/10.1016/j.burn.2014.03.003

[24] Garrett, S.K. and Kashawneh, M.T. (2011) Human Factors and Ergonomics in Healthcare Delivery: A Special Issue on Health Information Technology and Medication Administration Safety. International Journal of Industrial Ergonomics, 41, 333-335. https://doi.org/10.1016/j.ergon.2011.05.003

[25] Arndt, B.G., Beasley, J.W., et al. (2017) Tethered to the EHR: Primary Care Physician Workload Assessment Using EHR Event Log Data and Time-Motion Observations. The Annals of Family Medicine, 15, 419-426. https://doi.org/10.1370/afm.2121

[26] Amin, R.P. and Johansen, M.E. (2017) Tethered to the EHR: A Physician Workload Assessment. Annals of Family Medicine, 15, iii. https://doi.org/10.1370/afm.2147

[27] Sinsky, C., Colligan, L., et al. (2016) Allocation of Physician Time in Ambulatory Practice: A Time and Motion Study in 4 Specialties. Annals of Internal Medicine, 165, 753-760. https://doi.org/10.7326/M16-0961

[28] Dawson, D. and Reid, K. (1997) Fatigue, Alcohol and Performance Impairment. Nature, 388, 235. https://doi.org/10.1038/40775

[29] Dyrbye, L.N., Sotile, W., et al. (2014) A Survey of U.S. Physicians and Their Partners Regarding the Impact of Work-Home Conflict. Journal of General Internal Medicine, 29, 155-161. https://doi.org/10.1007/s11606-013-2581-3

[30] Henrikson, K., Dayton, E., et al. (2008) Chapter 5. Understanding Adverse Events: A Human Factors Framework. In: Hughes, R.G., Ed., Patient Safety and Quality: An Evidence-Based Handbook for Nurses, Agency for Healthcare Research and Quality (US), Rockville, MD.

[31] Frieden, T. (2010) A Framework for Public Health Action. The Health Impact Pyramid. American Journal of Public Health, 100, 590-595. https://doi.org/10.2105/AJPH.2009.185652

[32] Lowe, C.M. (2006) Accidents Waiting to Happen: The Contribution of Latent Conditions to Patient Safety. BMJ Quality \& Safety, 15, i72-i75. https://doi.org/10.1136/qshc.2006.016071

[33] Croskerry, P., Abbass, A. and Wu, A.W. (2010) Emotional Influences in Patient Safety. Journal of Patient Safety, 6, 199-205. https://doi.org/10.1097/PTS.0b013e3181f6c01a

[34] Arnsten, A.F.T. (1998) The Biology of Being Frazzled. Neuroscience, 280, 1711-1712. 
https://doi.org/10.1126/science.280.5370.1711

[35] Glavin, R.J. (2011) Human Performance Limitations (Communication, Stress, Prospective Memory and Fatigue). Best Practice \& Research: Clinical Anaesthesiology, 25, 193-206. https://doi.org/10.1016/j.bpa.2011.01.004

[36] Engel, G. (1980) The Clinical Application of the Biopsychosocial Model. American Journal of Psychiatry, 137, 535-544. https://doi.org/10.1176/ajp.137.5.535

[37] Heatherton, T.F. and Wagner, D.D. (2011) Cognitive Neuroscience of Self-Regulation Failure. Trends in Cognitive Sciences, 15, 132-139. https://doi.org/10.1016/j.tics.2010.12.005

[38] Pickering, B.W., Herasevich, V., Ahmed, A. and Gajic, O. (2010) Novel Representation of Clinical Information in the ICU. Applied Clinical Informatics, 1, 116-131. https://doi.org/10.4338/ACI-2009-12-CR-0027

[39] Michel, A. (2016) Burnout and the Brain. Association for Psychological Science. http://www.psychologicalscience.org/index.php/publications/observer/2016/februar y-16/burnout-and-the-brain.html

[40] Savic, I. (2015) Structural Changes of the Brain in Relation to Occupational Stress. Cerebral Cortex, 25, 1554-1564. https://doi.org/10.1093/cercor/bht348

[41] Golkar, A., et al. (2014) The Influence of Work Related Chronic Stress on the Regulation of Emotion and Functional Connectivity in the Brain. PLOS ONE, 9, e104550. https://doi.org/10.1371/journal.pone.0104550

[42] NIOSH (2008) Exposure to Stress: Occupational Hazards in Hospitals. Centers for Disease Control and Prevention, Publication No. 2008-136, 1-13.

https://www.cdc.gov/niosh/docs/2008-136/

[43] Alkadhi, K. (2013) Brain Physiology and Pathophysiology in Mental Stress. ISRN Physiology, 2013, Article ID: 806104.

https://www.hindawi.com/journals/isrn/2013/806104/ https://doi.org/10.1155/2013/806104

[44] Ridout, K.K., Ridout, S.J., Guille, C., Mata, D.A., Akil, H. and Sen, S. (2019) Physician Training Stress and Accelerated Cellular Aging. Biological Psychiatry, in press. https://doi.org/10.1016/j.biopsych.2019.04.030

[45] Wurm, W., Vogel, K., et al. (2016) Depression-Burnout Overlap in Physicians. PLoS ONE, 11, e0149913. https://doi.org/10.1371/journal.pone.0149913

[46] Risken, A., Erez, A., et al. (2015) The Impact of Rudeness on Medical Team Performance: A Randomized Trial. Pediatrics, 136, 487-495. https://doi.org/10.1542/peds.2015-1385

[47] Hadley, A., Adair, K., Rehder, K. and Sexton, J. (2018) Disruptive Behaviors in Healthcare Setting and Their Effect on Safety Culture. Critical Care Medicine, 47, 40. https://doi.org/10.1097/01.ccm.0000550871.12369.6c

[48] Ariely, D., Gneezy, U., Lowenstein, G., et al. (2005) Large Stakes and Big Mistakes. Federal Reserve Bank of Boston Working Paper No. 05-11.

[49] Dan Pink (2010) The Surprising Truth about What Motivates Us. https://www.youtube.com/watch?v=u6XAPnuFjJc

[50] Privitera, M.R. (2018) Is Burnout a Form of Depression? It's Not That Simple. Medscape Psychiatry, Requested Commentary. https://www.medscape.com/viewarticle/896537

[51] Stahl, S.M. (2000) Essential Psychopharmacology: Neuroscientific Basis and Practical Applications. 2nd Edition, Cambridge University Press, Cambridge, UK, 152.

[52] Kaplan, H.I. and Sadock, B.J. (1998) Synopsis of Psychiatry: Behavioral Sciences, 
Clinical Psychiatry. 8th Edition, Chapter 3, Lippincott Williams \& Wilkins, Baltimore.

[53] Nemeroff, C. (1998) The Neurobiology of Depression. Scientific American, 278, 42-49. https://doi.org/10.1038/scientificamerican0698-42

[54] Frazer, A. (2000) Norepinephrine Involvement in Antidepressant Action. Journal of Clinical Psychiatry, 61, 25-30.

[55] Martin, R. (2007) How Successful Leaders Think. Harvard Business Review, 85, 60-67.

[56] Dimoka, A. and Banker, R.V., et al. (2012) On the Use of Neurophysiological Tool in IS Research: Developing a Research Agenda for NeuroIS. MIS Quarterly, 36, 679-702.

[57] Privitera, M.R. (2018) Addressing Human Factors in Burnout and the Delivery of Healthcare: Quality \& Safety Imperative of the Quadruple Aim. Health, 10, 629-644. https://doi.org/10.4236/health.2018.105049

[58] Privitera, M.R., Plessow, F. and Rosenstein, A.H. (2015) Burnout as a Safety Issue: How Physician Cognitive Workload Impacts Care. National Patient Safety Foundation e-News.

https://www.npsf.org/blogpost/1158873/224974/Burnout-as-a-Safety-Issue--How-P hysician-Cognitive-Workload-Impacts-Care?tag=burnout

[59] Sweller, J. (1988) Cognitive load During Problem Solving: Effects on Learning. Cognitive Science, 12, 257-285. https://doi.org/10.1207/s15516709cog1202_4

[60] Berwick, D.M., Nolan, T.W. and Whittington, J. (2008) The Triple Aim: Care, Health and Cost. Health Affairs (Millwood), 27, 759-769. https://doi.org/10.1377/hlthaff.27.3.759

[61] Fleming, C. (2010) Berwick Brings “The Triple Aim” to CMS. Health Affairs Blog. https://www.healthaffairs.org/do/10.1377/hblog20100914.006950/full/

[62] Spinelli, W.M. (2013) The Phantom Limb of the Triple Aim. Mayo Clinic Proceedings, 88, 1356-1357. https://doi.org/10.1016/j.mayocp.2013.08.017

[63] Bodenheimer, T. and Sinsky, C.A. (2014) From Triple to Quadruple Aim: Care of the Patient Requires Care of the Provider. The Annals of Family Medicine, 12, 573-576. https://doi.org/10.1370/afm.1713

[64] Sikka, R., Morath, J.M. and Leape, L. (2015) The Quadruple Aim: Care, Health, Cost and Meaning in Work. BMJ Quality \& Safety, 24, 608-610. https://doi.org/10.1136/bmjqs-2015-004160

[65] Weisz, J.R., McCabe, M.A. and Dennig, M.D. (1994) Primary and Secondary Control among Children Undergoing Medical Procedures: Adjustment as a Function of Coping Style. Journal of Consulting and Clinical Psychology, 62, 324-332. https://doi.org/10.1037/0022-006X.62.2.324

[66] Jones, J.W., Barge, B.N., et al. (1998) Stress and Medical Malpractice: Organizational Risk Assessment and Intervention. Journal of Applied Psychology, 73, 727-735. https://doi.org/10.1037/0021-9010.73.4.727

[67] Fahrenkopf, A.M., Sectish, T.C., et al. (2008) Rates of Medication Errors among Depressed and Burnt out Residents: Prospective Cohort Study. BMJ, 336, 488-491. https://doi.org/10.1136/bmj.39469.763218.BE

[68] West, C.P., Huschka, M.M., et al. (2006) Association of Perceived Medical Errors with Resident Distress and Empathy. JAMA, 296, 1071-1078.

https://doi.org/10.1001/jama.296.9.1071

[69] Linzer, M., Poplau, S., et al. (2015) A Cluster Randomized Trial of Interventions to 
Improve Work Conditions and Clinician Burnout in Primary Care: Results from the Healthy Work Place (HWP) Study. Journal of General Internal Medicine, 30, 1105-1111. https://doi.org/10.1007/s11606-015-3235-4

[70] Simpson, K.R. and Knox, G.E. (2003) Recognizing and Understanding Adverse Perinatal Outcomes and Preventing Common Accidents. AWHONN Lifelines, 7, 224-235. https://doi.org/10.1177/1091592303255715

[71] Nolan, T.W. (2000) Education and Debate: System Changes to Improve Patient Safety. BMJ, 320, 771-773. https://doi.org/10.1136/bmj.320.7237.771

[72] https://www.ny.gov/combating-sexual-harassment-workplace/workers

[73] Simon, H.A. (1956) Rational Choice and the Structure of the Environment. Psychological Review, 63, 129-138. https://doi.org/10.1037/h0042769

[74] Berwick, D.M., Loehrer, S. and Gunther-Murphy, C. (2017) Breaking the Rules for Better Care. JAMA, 317, 2161-2162. https://doi.org/10.1001/jama.2017.4703

[75] Merlino, J. (2015) The Responsibility Matrix: A Strategy for Stronger Physician/Administrator Partnerships. Becker's Hospital Review.

https://www.beckershospitalreview.com/hospital-physician-relationships/the-respo nsibility-matrix-a-strategy-for-stronger-physician-administrator-partnerships.html 